\title{
DIE WAHRNEHMUNG DER FREMDSPRACHEN UND SPRACHBEWUSSTHEIT IM KINDESALTER
}

\begin{abstract}
Aysel Uzuntaş
Zusammenfassung

In dem Aufsatz handelt es sich um Fremdsprachenlernen und Sprachbewusstheit im Kindesalter. Es werden im außerschulischen Kontext anhand von spontanen Beobachtungen eines Kindes -in seinem Lebensalter zwischen sieben und acht Jahren- beschrieben, wie Fremdsprachen (Deutsch, Englisch) wahrgenommen und erlernt werden. Es stellt sich heraus, dass die Bedeutungserschließung und das Erfassen des Systems der Fremdsprache sich zunächst mit Hilfe der Muttersprache (Türkisch) vollziehen. Das Rätseln, das Entdecken der Bedeutungen von Äußerungen wie das Vergleichen von Sprachen spielen eine große Rolle beim Fremdsprachenlernen und tragen zur Sprachaufmerksamkeit bzw. -bewusstheit bei, die eine sprachfördernde Funktion ausüben.
\end{abstract}

Schlüsselwörter: Fremdsprache, Kindesalter, Wahrnehmung, Sprachvergleich, Sprachbewusstheit.

\section{ERKEN YAŞTA YABANCI DILLLERIN ALGILANMASI VE DÍL BÍLINCI}

\begin{abstract}
$\ddot{O z e t}$
Bu makalede erken yaşta yabancı dil ögrenimi ve dil bilinci ele alınmaktadır. 7-8 yaş aralı̆̆ındaki bir çocuğun okul dışında gözlemlenmesi ve onun yabancı dile yaklaşımı, yabancı dili algılama ve öğrenme biçimi betimlenmekte ve değerlendirilmektedir. Anlam çıkarma ve yabancı dilin sisteminin çözümlenmesinin öncelikle anadil yardımılla gerçekleştiği tespit edilmektedir. Sözlerin anlamlarının keşfedilmesi, dillerin karşılaştırılması yabancı dil öğretiminde önemli rol oynamakta ve dilde farkındalık ve dil bilincinin oluşmasına katkıda bulunmaktadır.
\end{abstract} Anahtar Kelimeler: Yabancı dil, erken yaş, algl, dil karşılaştırması, dil bilinci. 


\section{EINLEITUNG}

Der Frühbeginn gewinnt seit den 90'er Jahren an Bedeutung. Nicht zuletzt wird im Rahmen der Sprachenpolitik der EU, in der es um Mehrsprachigkeit geht, für das frühe Fremdsprachenlernen plädiert, da das Lernen von mindestens zwei Fremdsprachen beabsichtigt wird (vgl. Edelenbos/ Kubanek 2007).

„Es wird weithin angenommen, dass Alter als wichtiger Einflussfaktor beim Sprachenlernen gilt. Mit steigendem Alter lasse die Fähigkeit zum Sprachenlernen nach. In jüngeren Jahren falle dagegen das Sprachlernen leichter, weil es spielerisch erfolge. (...)“" (Roche 2005: 37).

Diverse Faktoren sprechen für das Fremdsprachenlernen im Kindesalter. Es stellt sich jedoch die Frage, warum Kinder Fremdsprachen lernen, denn Erwachsene haben viele Gründe und Ziele beim Fremdsprachenlernen wie das Studium, der Beruf, persönliche Kontakte usw., und Kinder? Ist das überhaupt deren eigene Entscheidung eine fremde Sprache zu lernen? Wie lernen sie sie, wenn sie im Unterschied zu den Erwachsenen nicht zielorientiert verfahren? Wie werden Fremdsprachen von den Kindern wahrgenommen? Sind Kinder aufmerksam auf die Fremdsprachen? Es ist daher wichtig zu ermitteln, was für ein Interesse die Kinder für die Fremdsprache zeigen, die mit der Fremdsprache konfrontiert werden und gefördert werden, sie zu erlernen

Wenn man an die Kinder denkt, die ,eine fremde Sprache' als Zweitsprache erwerben, kann man den Erwerb der ,fremden' Sprache (L2) nachvollziehen, da diese Kinder die zweite Sprache, die ihnen zunächst fremd ist, im natürlichen Kontext lernen bzw. erwerben. Sie sind mit der Zweitsprache in ihrem sozialen Umfeld konfrontiert. In dieser Hinsicht deutet auch Kiehlhöfer (1989) auf die soziale Perspektive des Sprachlernens hin: „Kinder erwerben eine Sprache, weil sie sie sozial brauchen" (ebd.: 360). Hier ist zwischen der Fremdsprache und Zweitsprache zu unterscheiden, da die Kinder aufmerksam auf die Zweitsprache sind, die sie in ihrer Umgebung stets hören, und die sie für die Kommunikation im Alltag gebrauchen. Im Zusammenhang mit der Zweitsprache sollte die Sprachaufmerksamkeit auch beim Fremdsprachenlernen gefördert werden, auf die Oomen-Welke (2000) und Edelenbos/ Kubanek (2007) auch hindeuten: „Die Begeisterung der Kinder und ihre Offenheit gegenüber anderen Sprachen und Kulturen müssen aktiv gefördert und dürfen nicht einfach dem Zufall überlassen werden“ (Edelenbos/ Kubanek: 26).

Der vorliegende Aufsatz möchte anhand von Spontanbeobachtungen und durch Beispiele darstellen, wie Kinder Fremdsprachen wahrnehmen und was für eine Bedeutung sie für sie haben. Die Beobachtung wird im außerschulischen Kontext durchgeführt, da das Fremdsprachenlernen im schulischen Kontext ein gesteuerter 
Prozess ist, wobei hier festgestellt werden möchte, wie Kinder im natürlichen Kontext auf die Fremdsprachen reagieren.

Die Beobachtung eines Kindes, die in diesem Aufsatz thematisiert wird, beruht auf einer Langzeitbeobachtung im Alltagsleben. Die Beobachtungen sind Spontanbeobachtungen, die dokumentiert wurden. Sie werden in diesem Aufsatz deskribiert, indem induktiv vorgegangen wird. Aus diesen Beobachtungen wird versucht, diverse Perspektiven des Fremdsprachenlernens darzulegen. Es ist ein wichtiges Thema, wie Kinder überhaupt die Fremdsprachen wahrnehmen, bewerten und darüber hinaus, wie sie sie versuchen in sich zu integrieren und zu erklären.

\section{Die Wahrnehmung der Fremdsprachen}

Was bedeutet für die Kinder, dass eine Sprache ,fremd' ist?

Auf die Frage, welche Bedeutung die Fremdsprache für Kinder hat, wird hier versucht im Rahmen der spontanen Beobachtungen eines Kindes - in seiner Entwicklung zwischen dem Lebensalter von 7 bis 8 Jahren - einzugehen. Die Beobachtungen deuten auch auf den Unterschied hin, wie Kinder mit der Zeit, die Fremdsprache anders wahrnehmen und bewerten. Hier wird von einem Jungen, Ali ${ }^{1}$, berichtet, der im Alltagsleben für eine lange Zeit spontan beobachtet wurde, und dadurch ermittelt werden konnte, welche Reaktionen er zur Fremdsprache in einer außerschulischen, authentischen Situation gibt.

Ali hat schon von Kleinheit an Wörter auf Deutsch gehört, da in seiner Umgebung in der Türkei ab und zu deutsche Wörter gesprochen wurden. Seine Mutter und seine Tanten sind Rückkehrerkinder aus Deutschland. Während die Mutter von Ali Deutsch nicht aktiv beherrscht, aber einiges verstehen kann, sprechen die beiden Tanten fließend Deutsch. Ali war somit sehr interessiert für Deutsch, weil in seiner Gegenwart, mit Zeitabständen und nicht intensiv, auch Deutsch gesprochen wurde, vor allem, wenn deutsche Gäste und Verwandte aus Deutschland mit zeitlich großen Abständen zu Besuch in der Türkei waren, war er mit Deutsch konfrontiert. So wollte er von selbst mit sieben Jahren auch Deutsch verstehen und sprechen können. Er war sehr motiviert und bereit diese Sprache zu verstehen und zu sprechen, da er diese Sprache, wie Kielhöfer (1989) es betont, in bestimmten Situationen und Zeiten sozial brauchte, um das Gesprochene, das ihm fremd war, zu verstehen.

Am Anfang bezeichnete Ali mit sieben Jahren Deutsch als eine Rätselsprache und wollte diese ,Sprache als Rätsel' lernen, d.h. er wollte erraten und mit dieser Sprache spielen. Er äußerte folgendes: „Bilmece dilini konuşsana benimle“ („Sprich mit mir diese Rätselsprache“). Diese Bezeichnung der Fremdsprache als

1 Der Name des Kindes ist verändert worden als Ali. 
Rätselsprache ist sehr interessant und auch berechtigt. Ali beschrieb die ihm fremde Sprache als Rätselsprache ${ }^{2}$, da er es erkannte, dass die Wörter eine Bedeutung haben und etwas darstellen wie die Muttersprache, aber er erkannte es auch, dass diese doch nicht wie die Muttersprache waren. Er erklärte diese ihm fremde Sprache vom Zentrum seiner Muttersprache. Er ging stets von seiner Muttersprache Türkisch aus und wusste von der Sprachenvielfalt unserer Welt nichts, denn in seiner Umgebung gab es nur eine Sprache, die Muttersprache. Die deutschen Wörter, die er ab und zu hörte, waren für ihn etwas, das gerätselt wird. Deutsch war für ihn ein Rätsel, das er in einem bestimmten Kontext lösen konnte, erraten konnte und es gefiel ihm sehr, indem er die Bedeutungen der Wörter, Sätze herausfand.

Nachdem Ali die Fremdsprache Deutsch als ,Rätselsprache' definierte, wurde es ihm versucht die Sprachenvielfalt unserer Welt zu erklären und ihn darüber bewusst zu machen, dass es nicht nur die Muttersprache Türkisch gibt, sondern auch viele andere Sprachen, die auch Muttersprachen von anderen sind. Während andere Sprachen wie Deutsch, Englisch für Ali eine Fremdsprache sind, ist Türkisch für andere, wie z.B. für deutschsprachige, englischsprachige Muttersprachler eine Fremdsprache. Obwohl Ali dies zunächst nicht ganz verstehen konnte, weil es für ihn die Muttersprache Türkisch und die ,Rätselsprachen' gaben, begriff er die Vielsprachigkeit der Welt, indem auch ein Globus zur Hilfe genommen wurde, um zeigen zu können, welche Sprachen auf der Welt gesprochen werden.

Auch wenn das Kind Bedeutungen aus der ihm fremden Sprache erschließen kann, trägt die sprachliche Sensibilisierung und Bewusstmachung über die Sprachenvielfalt auch zu einem Perspektivenwechsel bei. Sprachen wie Deutsch und Englisch sind aus der Perspektive von Ali Fremdsprachen, aber aus der Perspektive von deutschsprachigen, englischsprachigen Muttersprachlern sind sie keine Fremdsprachen, sondern Muttersprachen wie Türkisch. Es gibt auf der Welt diverse Muttersprachen und Fremdsprachen.

\section{Wie können Kinder Sprachen voneinander unterscheiden?}

Kinder sind sehr gute Sprachanalytiker, die auf verschiedener Weise über die Sprache reflektieren können. Oomen-Welke (2000) deutet darauf hin, wie wichtig die Sprachaufmerksamkeit beim Sprachlernen ist:

„Aufmerksamkeit auf Sprachliches begleitet das sprachliche Lernen und oft auch anderes Erfahren und Lernen. Kinder werden sehr früh auf Sprachliches aufmerksam, besonders wenn sie in sprachlich anregender Umgebung aufwachsen“(Oomen-Welke 2000: 147).

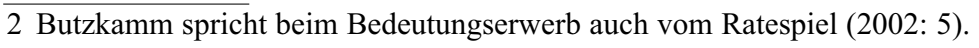


So ist auch Ali auf die Sprachen sehr aufmerksam und kann nicht nur zwischen Türkisch und Deutsch differenzieren, sondern auch zwischen Deutsch und Englisch, die er beide nicht beherrscht. Wenn mit ihm Deutsch und Englisch gesprochen wurde, damit er erraten konnte, was ihm gesagt wurde, verstand er, ob es sich um die englische oder deutsche Sprache handelte. Auf die Frage, wie er zwischen diesen beiden Sprachen differenzieren konnte, machte er metasprachliche Erklärungen. Er erklärte, dass die deutsche Sprache ein langsames Tempo hat, wogegen das Englische ein schnelles Tempo hat. Auf die Eigenschaft der Intonation, der Sprachmelodie in beiden Sprachen ist das Kind aufmerksam und kann Sprachen diesbezüglich voneinander unterscheiden. Das zeigt auch, wie wichtig die Sprachmelodie, d.h. die lautliche Seite der Sprache ist. Auch wenn der Inhalt nicht bekannt ist, ist die Ausdrucksseite zunächst ein wesentlicher Indikator der Sprachen. Zunächst hört das Kind und erschließt das System der Sprache. Wo fängt ein Wort an und wo hört es auf, was für ein Sprachtempo hat die Sprache u. a. Diese paraverbalen Signale der Sprachen sind für die Wahrnehmung der Fremdsprachenlerner von besonderer Relevanz und dienen auch der Sprachsensibilisierung. Diese Sprachsensibilisierung kann dadurch gefördert werden, dass mit den Kindern erstmal in der Fremdsprache gesprochen wird und auch, dass daraus ein Spiel gemacht wird wie z.B.: „Errate um welche Sprache es sich jetzt handelt. Deutsch oder Englisch?“. Ali macht es viel Spaß Sprachen anhand von Kriterien, die er selbst bestimmt, voneinander unterscheiden zu können. Das ist für ihn ein Spiel, das er stets spielen möchte. Sprachen sind wie Lieder und verfügen über eigene Melodien. Für die Schulung können zwei oder drei Sprachen hintereinander gesprochen werden, damit das Kind auf die lautlichen Differenzen aufmerksam wird. Ali ist aber nicht nur auf die lautliche Seite der Sprachen aufmerksam, sondern auch auf die Struktur der Sprachen. In Bezug auf die Muttersprache versucht Ali die Struktur der Fremdsprache zu verstehen. Dieser Verstehensprozess wird im Vergleich mit der Muttersprache vollzogen.

Es stellt sich heraus, dass es sehr relevant ist bei den Kindern zunächst die Sprachaufmerksamkeit zu fördern. Wenn das Kind aufmerksam auf die Sprache ist, fängt es an, sich auch für die Fremdsprache zu interessieren und auch Mühe geben, die Fremdsprache zu erlernen. Durch Sprachvergleiche sowohl lautlicher als auch inhaltlicher Art werden Kinder sprachbewusster und können über die Sprachen reflektieren. So macht Ali sprachliche und metasprachliche Erklärungen über die Sprachen, mit denen er konfrontiert wird.

Wie erschließen die Kinder Bedeutungen einer Fremdsprache?

Auch wenn Ali der Fremdsprache Deutsch nicht mächtig ist, kann er Bedeutungen erschließen. Er kann aus dem Kontext erraten, was gesagt wird. Butzkamm (2002) 
spricht im Rahmen des Mutterspracherwerbs von „Bedeutungserwerb als ein Ratespiel, in dem sich das Kind auf das Gesagte konzentriert und seine ganze Intelligenz einsetzt, um dessen Sinn zu erschließen“ (ebd.: 5). Dies ist bei der vorliegenden Beobachtung auch bei der Bedeutungserschließung in der Fremdsprache zu ermitteln. Für Ali sind der Kontext und die nonverbalen Anzeichen eine Hilfe für die Erschließung der Bedeutung. So sind die Situationen authentisch wie beim Erwerb der Muttersprache. Knapp (2007) plädiert auch dafür, dass „Sprachförderung möglichst nahe an realen Interaktionen ausgerichtet sein [sollte]“ (ebd.: 186) und deutet in Bezug auf den Begriffserwerb bei den Kindern auf folgendes hin:

„Die Dominanz der Kärtchen und Bilder bei der Sprachförderung verhindert die Aneignung differenzierter Bedeutungen genauso wie den Erwerb der relevanten syntaktischen und morphologischen Muster, ganz zu schweigen von Textschemata“" (ebd.: 186).

Wie kann beim Fremdsprachenlernen Authentizität verschaffen werden und wie sind realitätsnahe Interaktionen darzustellen?

Außer dem Erraten der Bedeutung im Kontext und in authentischen Situationen, die ihm bekannt sind, machte es Ali viel Spaß deutsche Sendungen anzuschauen, obwohl er die Sprache nicht beherrschte. Er konnte in den Sendungen die ihm vertrauten Wörter herausfinden und daraus im Sinnzusammenhang lernen. In einer Kindersendung z.B. kam der Satz "Gib mir eine ..." vor, da schrie er: "Gib mir". Er erkannte diese "Gib mir..." Konstruktion, die ihm bekannt war und konnte somit verstehen, dass es sich um das Geben handelte. Nicht nur das Verstehen machte ihn glücklich, er wurde auch dadurch motiviert, dass er rätseln konnte, Bedeutungen erschließen konnte. Somit schaffte er etwas. Ihm machte es viel Freude, aus dem ihm Unverständlichen etwas verstehen zu können. Das war für ihn eine Art Spiel und ein Erfolg des Entdeckens. Ferner erschloss das Kind in den deutschen Sendungen die Internationalismen mit großer Motivation, da er dadurch die ihm fremde Sprache verstand.

Neben dem kontextgebundenen Verstehen konnte Ali auch schon einiges auf Deutsch sprechen wie "Gib mir bitte Wasser", "Gib mir ein Küsschen", "Ich möchte Wasser", da er immer nachfragte und wissen wollte, wie man die oben geschilderten Redemittel -als Entsprechungen für die türkischen Redemittel- und Sprechakte (Bitten: Geben, Möchten) auf Deutsch ausdrückt.

Wie es aus den Beispielen auch ersichtlich ist, sind diese Sätze im Imperativ oder mit Modalverben, die in einem Lehrwerk erst in den späteren Kapiteln vorkommen. Ali zeigte kein Interesse zu den, in den Lehrwerken am Anfang stehenden Vorstellungen "Wie heißt du? Ich heiße ...", weil diese für ihn unter uns nicht 
authentisch und real waren, da wir unsere Namen schon wussten. Er wollte andere Sachen ausdrücken, die für ihn von großer Bedeutung sind, und diese fangen vielmals mit "Ich möchte ..." oder "Gib mir bitte ..." an. Das macht auch ersichtlich, dass die Welt des Kindes, seine Interessen und Bedürfnisse im Vordergrund stehen.

Bei dem Verstehensprozess und beim Sprechen in der Fremdsprache machte Ali von selbst Vergleiche mit der Muttersprache, die im Folgenden behandelt werden.

\section{Der Bezug auf die Muttersprache - Sprachvergleiche zur Sprachbewusstheit}

Nachdem das Kind (Ali) begriffen hatte, dass es mehrere Sprachen auf der Welt gibt, und dass die einzige Sprache nicht Türkisch ist, ging es immer sprachbewusster auf die Sprachen ein. Mit acht Jahren hat er angefangen über die Sprachen besser zu reflektieren. Einerseits möchte er die Bedeutungen der Wörter anderer Sprachen wissen, andererseits macht er auch von selbst Vergleiche mit der Muttersprache. Der Titel eines deutschsprachigen Buches „Was ist Kunst”, das er im Bücherregal entdeckt, erweckt sein Interesse und macht ihn auch aufmerksam. Er fragt, was das auf Türkisch heißt. „Was ist Kunst“ heißt „Sanat nedir”. Er begnügt sich nicht mit der Übersetzung, er , analysiert' die Sprachen und meint, dass es im Deutschen drei Wörter gibt, im Türkischen dagegen zwei Wörter. Ferner versucht er die beiden Entsprechungen zu erklären und meint, dass dann das Wort ,was' , sanat 'heißt. Er bekommt es erklärt, dass ,Kunst' , sanat' heißt. Er deutet darauf hin, dass dann das Wort Kunst im Deutschen zuletzt, im Türkischen dagegen zuerst vorkommt und fragt, welche Entsprechung dann ,wer' hat. Auf die Antwort ,ne'hat er immer noch Fragen im Kopf. Denn wo bleibt dann die Entsprechung von ,ist' im Deutschen, und es heißt ja nicht ,sanat ne', sondern ,sanat nedir'. Warum ist die Entsprechung von ,was' nicht ,nedir', sondern ,ne'? Beide Sprachen haben ein unterschiedliches System. Die Wortstellungen sind anders, ferner auch die Zahl der entsprechenden Wörter in der einen oder anderen Sprache. Quantitativ können Ausdrücke nicht immer eine Eins-zu-eins Entsprechung haben.

(1) Was ist Kunst? $\rightarrow$ à drei Wörter im Deutschen $\begin{array}{lll}1 & 2 & 3\end{array}$

(2) Sanat nedir? $\rightarrow$ à zwei Wörter im Türkischen 12

Die türkischen Entsprechungen der drei Wörter im Deutschen sind folgende:

(3) was: ne, ist: -dir, Kunst: sanat. 
Das Kopulaverb sein entspricht im Türkischen dem Affix -dir. Deutsch und Türkisch sind typologisch unterschiedliche Sprachen. Ihre Satzstruktur, Wortstellungsregeln, ihre Flexionsformen sind anders, Deutsch ist eine flektierende, Türkisch eine agglutinierende Sprache.

Der acht Jährige versucht die Systeme beider Sprachen in sich zu integrieren und greift stets auf die Muttersprache zurück und versucht somit das für ihn Neue mit der vorhandenen Muttersprache zu erklären bzw. zu verstehen, obwohl er mit der Fremdsprache nicht intensiv konfrontiert wird. Er versucht insofern die ihm fremden Wörter und Strukturen in Bezug auf die Muttersprache sich anzueignen. Neben den Bedeutungen der Wörter stehen vor allem die Wortstellungen im Mittelpunkt seines Interesses.

Mit acht Jahren ist Ali auf alle Sprachen, mit denen er in irgendeiner Weise in Kontakt kommt, aufmerksam. Er achtet auf die Schilder in Restaurants. Er meint, er weiß, was ,smoking'im Englischen heißt, sigara içmek' und fragt dann, was das Schild ,No smoking' bedeutet. Es bedeutet ,sigara içilmez'. Er erklärt von selbst, dass dann ,no' ,içilmez' heißt, denn beide Sätze, im Englischen und im Türkischen, bestehen aus zwei Wörtern und müssten dann gleiche Entsprechungen haben. Aber ,smoking' hat im Türkischen als Entsprechung ein zusammengesetztes Verb ,sigara içmek', wortwörtlich heißt es ,Zigarette trinken'. ,No' entspricht in diesem Beispielsatz dem Negationssuffix -mez. Er erklärt dann wieder von selbst, als ein Versteh- und Aneignungsprozess (vgl. Wolff 1993), dass im Englischen ,smoking' am Ende, im Türkischen dagegen , sigara içmek' vorne steht.

(4) No smoking. $\rightarrow$ à zwei Wörter im Englischen 12

(5) Sigara içilmez $\mathrm{z}^{3} \rightarrow$ à zwei Wörter im Türkischen 12

Wie die Sätze (4) und (5) auch exemplifizieren, kann die quantitative Entsprechung der Wörter täuschen, da ein Wort in der einen Sprache durch zwei oder mehrere Wörter bzw. Gefügen ausgedrückt werden kann. Ferner ist der Sprachtyp der jeweiligen Sprachen auch bestimmend. Da das Türkische eine agglutinierende Sprache ist, entsprechen Wörter im Deutschen oder im Englischen nicht selten den Suffixen im Türkischen (ist : -dir; no : -mez).

Wie die obigen Beispiele es auch zeigen, macht das Kind stets Sprachvergleiche und versucht das Neue mit seinem vorherigen Wissen anzuknüpfen (vgl. Wolff

3 Bei der türkischen Entsprechung wird das Verb in passiver Form verwendet: içmek vs. içilmek. Auch diesbezüglich gibt es Differenzen. 
1993; Oomen-Welke 2000; Butzkamm 2002; Akdoğan 2007). So bezieht sich Ali immer wieder auf die Muttersprache und nimmt sie als eine Grundlage für das Verstehen der anderen Sprachen. Wolff (1993) spricht hier von „Aufbau von Sprachbewußtheit über Sprachkontrast" (ebd.: 523). Durch die Sprachvergleiche reflektiert das Kind auch über die eigene Muttersprache, da er das System der Muttersprache somit erschließt (vgl. ebd.: 527). Und das alles geschieht nicht nur aus lexikologischer, sondern auch aus syntaktischer Sicht. Die Anzahl der Wörter, die Reihenfolge der Wörter sind für das Kind besondere Merkmale, für die es eine direkte Entsprechung sucht.

Diese Beobachtungen und Erfahrungen weisen darauf hin, dass Ali sprachaufmerksam ist, da er im authentischen Kontext mit Wörtern in der fremden Sprache in Beziehung kommt. Er kann zwischen der Muttersprache und Fremdsprache wie auch zwischen den Fremdsprachen differenzieren. Er bewertet die Fremdsprache zunächst als ein Rätsel, da er von der Einsprachigkeit ausgeht und in dieser Art und Weise bereit ist, Sprachen zu erkennen, Bedeutungen zu erschließen. Das Erschließen von Wörtern in der fremden Sprache ist für ihn sehr motivierend. Er begnügt sich nicht nur mit dem Rezipieren, sondern möchte auch für ihn Wichtiges in der Fremdsprache ausdrücken können. Mit der Zeit lernt er über die Sprachen zu reflektieren und erfährt über die Mehrsprachigkeit der Welt. So wird er aufmerksamer und geht bewusster vor, indem er von selbst Sprachvergleiche macht und die Muttersprache als Basis zu Hilfe nimmt. In dieser Art und Weise versucht er das System der Fremdsprache zu erfassen und sich anzueignen. Seine Erklärungen sind nicht nur wörtliche Bedeutungserklärungen bzw. wörtliche Übersetzungen; er macht auch syntaktische Erklärungen, indem er sein Augenmerk auf die Entsprechungen der Anzahl der Wörter in einem Satz in der Fremdsprache und der Muttersprache sowie auf die Wortstellung, Reihenfolge der Wörter in Sprachen richtet.

\section{Ausblick}

Auch wenn in diesem Aufsatz von spontanen Beobachtungen anhand eines Kindes ausgegangen worden ist, und diese Beobachtungen nicht immer verallgemeinert werden können, da auch viele Variablen vorhanden sind, geben die Beobachtungen viele Impulse zum Fremdsprachenlernen im Kindesalter und zur Relation zwischen Muttersprache und Fremdsprache, die m. E.. sowohl für den Muttersprachenunterricht als auch für den Fremdsprachenunterricht im Rahmen der Förderung der Sprachaufmerksamkeit bzw. -bewusstheit angewandt werden können, da diese Beobachtung auch eine unter vielen Variablen ist und diesbezüglich auch zu beachten ist.

Induktiv kann man aus den Beobachtungen für das Fremdsprachenlernen im Kindesalter folgendes folgern: Die Beobachtungen zeigen, dass Kinder die 
Sprachen in unterschiedlicher Art und Weise wahrnehmen. Fremdsprache wird als Rätsel bezeichnet, da sie von der Muttersprache differenziert wird, da sie nicht zu der Sprache gehört, die beherrscht wird. Die Fremdsprache wird als Rätsel bezeichnet, da die Bedeutung der Wörter in der Fremdsprache gerätselt wird, und zwar kontextgebunden erraten bzw. entdeckt wird. Kinder erkennen über ihre Muttersprache hinaus andere Sprachen, in ihrer Sprachmelodie und Intonation und in ihrer Bedeutung von Wörtern. Sie begnügen sich nicht nur mit dem Erschließen der Bedeutung von Wörtern, sondern versuchen die Fremdsprache bezogen auf ihre Muttersprache zu verstehen und zu erklären. So sind folgende Besonderheiten beim Fremdsprachenlernen von eminenter Bedeutung:

- Förderung der Sprachaufmerksamkeit, Sprachbewusstheit.

- Erkennen von bereits vorhandenen Wissen und sie umsetzen können (Muttersprache, Sprachvergleiche).

- Authentizität verschaffen (Titel von Büchern, Schilder in Restaurants usw.).

- Eigene Interessen der Kinder berücksichtigen (Gib mir ..., ich möchte ...).

- Spaß beim Lernen haben, rätseln, mit der Sprache spielen und somit Sprachen lautlich unterscheiden können und ferner die Bedeutungen erraten können.

- Motivation erhöhen (sprachanregende Situationen verschaffen, etwas Unverständliches verstehen wollen, das Unverständliche verstehen können, sich in der fremden Sprache ausdrücken können. Die Freude des Entdeckens, des Könnens verschaffen).

- Fernsehsendungen in der fremden Sprache als Herausforderung für Kinder. Kinder, die aufmerksam sind, können in der fremden Sprache die Wörter, die ihnen vertraut sind, herausfinden und besser verarbeiten.

- Die Internationalismen verwenden, um auch Gemeinsamkeiten zwischen der vertrauten Muttersprache und der ,fremden' Sprache aufzuzeigen und eine Verbindung zwischen den Sprachen herzustellen.

Die kleinen Sprachanalytiker wollen erraten, entdecken, mit der Sprache experimentieren, wollen Spaß am Lernen haben. Diese Faktoren sollten auch im Fremdsprachenunterricht berücksichtigt werden. So sollte auch beim Fremdsprachenlernen im Unterricht der Muttersprache Beachtung geschenkt werden, damit die Kinder vergleichend vorgehen und mit den Sprachen auch spielend, entdeckend lernen können.

Auf die eingangs gestellte Frage, warum Kinder überhaupt Fremdsprachen lernen, da sie nicht selbst entscheiden zielorientiert eine Fremdsprache zu erlernen, kann fol- 
genderweise geantwortet werden, dass Kindern das Entdecken, Entschlüsseln des Fremden, das Rätseln von Bedeutungen große Freude macht, und dass sie dadurch auch Lust haben mit der Fremdsprache zu spielen, indem sie sie auch mit der Muttersprache, der sie mächtig sind, in Beziehung setzen. Sie suchen Entsprechungen in der Muttersprache, nicht nur inhaltlicher Art, d.h. sie suchen nicht nur nach Bedeutungsentsprechungen, sondern gehen auch logisch und mathematisch vor und suchen auch nach Entsprechungen in Anzahl von Wörtern in Aussagen und deren Reihenfolge im Satz. Für Kinder sind authentische Situationen nötig, da sie das Gesagte verstehen wollen und die Sprache für die Interaktion brauchen.

Nur: Kinder müssen zunächst aufmerksam auf die Fremdsprache gemacht werden. Das Interesse der Kinder auf die Fremdsprache muss erweckt werden, wo die Sprachförderungen dann folgen, indem die Muttersprache, von der die Kinder ausgehen, nicht ausgeklammert wird, sondern im Zentrum steht als eine Unterstützung des Verstehens und Lernens.

\section{Literaturverzeichnis}

Akdoğan, Feruzan (2007), Thesen und Beispiele zum frühen Unterricht in der Fremdsprache Deutsch. Überlegungen zu einem zielgruppenorientierten Vorgehen im Deutschunterricht an den türkischen bilingualen Grundschulen. Frankfurt am Main: Peter Lang.

Butzkamm, Wolfgang (2002), Psycholinguistik des Fremdsprachenunterrichts. 3. neubearb. Aufl. Tübingen und Basel: A. Francke Verlag.

Edelenbos, Peter/ Kubanek, Angelika (2007), „Fremdsprachen- Frühbeginn: einzigartige Lernchancen nutzen. Zu den Ergebnissen der Studie EAC 89/04 für die Europäische Komission“. In: Frühes Deutsch 10/2007.

(http://www.goethe.de/mmo/priv/2454420-STANDARD.pdf (Stand: 31.05.2009)

Kiehlhöfer, Bernd (1989), „Frühkindlicher Bilingualismus“. In: Bausch, K. / Christ, H. / Hüllen, W. et al.(Hrsg.): Handbuch Fremdsprachenunterricht. Tübingen: Francke Verlag. 359-363.

Knapp, Werner (2007), „Wie Kinder Begriffe erwerben und welche Annahmen Erwachsene darüber haben“. In: Jost, R.; Knapp, W.; Metz, K. (Hrsg).: Fachwissenschaftliche und fachdidaktische Aspekte. Baltmannsweiler: Schneider. 173-187.

Oomen-Welke, Ingelore (2000), „Umgang mit Vielsprachigkeit im Deutschuntericht Sprachen wahrnehmen und sichtbar machen“. In: Deutsch lernen, 25 (2000) 2. 143-163.

Roche, Jörg (2005), Fremdsprachenerwerb Fremdsprachendidaktik. Tübingen und Basel: A. Francke Verlag.

Wolff, Dieter (1993), „Sprachbewußtheit und die Begegnung mit Sprachen“. In: Die Neueren Sprachen, 92 (1993) 6. 510-531. 